\title{
Effects of the combined administration of risedronate and menatetrenone on bone loss induced by tacrolimus in rats
}

\author{
Junkichi Kanda ${ }^{1}$, Megumi Furukawa ${ }^{2}$, Nobuo Izumo ${ }^{2}$, Taketoshi Shimakura ${ }^{3}$, \\ Noriaki Yamamoto ${ }^{3,4}$, Hideaki E. Takahashi ${ }^{3}$, Hiroyuki Wakabayashi, ${ }^{1, *}$ \\ ${ }^{1}$ Department of Clinical Pharmacotherapy, Faculty of Pharmaceutical Sciences, Niigata University of Pharmacy and Applied Life Sciences, \\ Niigata, Japan; \\ ${ }^{2}$ General Health Medical Center Yokohama University of Pharmacy, Yokohama, Japan; \\ ${ }^{3}$ Niigata Bone Science Institute, Niigata, Japan; \\ ${ }^{4}$ Division of Orthopedic Surgery, Niigata Rehabilitation Hospital, Niigata, Japan.
}

SUMMARY Tacrolimus, a calcineurin inhibitor, affects bone metabolism and increases the risk of fracture due to marked bone loss. Bisphosphonates increase the bone mineral density (BMD) in osteoporosis patients. Menatetrenone has less positive effects on BMD but reduces the risk of fracture by improving bone quality. In this study, we investigated the effectiveness of the combined administration of risedronate and menatetrenone against bone loss induced by tacrolimus. Wistar rats were divided into four groups: [1] control, [2] tacrolimus at $1.5 \mathrm{mg} / \mathrm{kg}$, [3] tacrolimus + risedronate at $1.0 \mathrm{mg} / \mathrm{kg}$, and [4] tacrolimus + risedronate + menatetrenone at $20 \mathrm{mg} / \mathrm{kg}$. After the drugs were administered for 4 weeks, bone histomorphometric analysis was performed and bone strength was evaluated using a threepoint bending method. BMD was measured using quantitative computed tomography. Tacrolimus significantly reduced the BMD and strength properties of the lower limb bones. These tacrolimusinduced decreases were suppressed by risedronate treatment. The combined administration of risedronate and menatetrenone more significantly improved bone strength properties than risedronate alone. Bone histomorphometric analysis revealed a significant increase in bone resorption with tacrolimus. Risedronate alone significantly suppressed the tacrolimus-induced increase in bone resorption but simultaneously reduced bone formation. On the other hand, the combined administration of risedronate and menatetrenone suppressed the tacrolimus-induced increase in bone resorption, in addition to the significant risedronate-induced decrease in bone formation. This study suggests that the combined administration of risedronate and menatetrenone improves bone strength in tacrolimustreated rats by preventing and ameliorating the risedronate-induced suppression of bone formation.

Keywords Bisphosphonate, menatetrenone, combined administration, bone loss, tacrolimus

\section{Introduction}

To further extend the healthy life expectancy in the super-aged societies of developed countries, fractures, which significantly reduce quality of life and vital prognosis in patients, should be prevented. Bone is a metabolically active organ that undergoes continuous remodeling, consisting of bone resorption by osteoclasts and bone formation by osteoblasts (1). An imbalance of bone remodeling induced skeletal disorders, such as osteopenia or osteoporosis (2,3). Aging and menopause are the main causes of bone fragility leading to fractures, whereas certain diseases and drugs significantly affect bone metabolism, resulting in an increased risk of fracture. We previously reported that tacrolimus, a calcineurin inhibitor, increased osteoclast activity, which regulates bone metabolism, and induced bone loss (4). To promote immunosuppressive therapy, which is essential for the prevention of rejection in recipients, prevention and treatment methods should be established using pharmacotherapy for tacrolimus-induced bone loss.

Bone strength is defined by bone mineral density (BMD) and bone quality, which refers to bone microstructure, bone turnover, micro-damage, and bone mineralization (5). Bisphosphonates, bone resorption inhibitors, are used worldwide for the treatment of osteoporosis. Bisphosphonates suppress bone resorption by inhibiting osteoclast activity $(6,7)$. Of note, bisphosphonates have been demonstrated to increase 
BMD in clinical studies $(8-11)$ and osteoporosis animal models (12-14). However, long-term administration of bisphosphonates may degrade bone strength by markedly suppressing bone remodeling (15-17) and increase the risk of developing atypical femoral fractures (18-21).

Menatetrenone, vitamin $\mathrm{K}_{2}$, acts as an essential coenzyme for the $\gamma$-carboxylation of glutamate residues in osteocalcin, a bone matrix protein $(22,23)$, and significantly reduces the risk of fracture $(24,25)$. As menatetrenone slightly increases BMD (26), its ability to reduce the risk of fracture is primarily explained by improved bone quality (27). Therefore, the combined administration of bisphosphonate and menatetrenone, which have different mechanisms of action on bone metabolism, may have beneficial effects on both BMD and bone quality, which determine bone strength, although its detailed effects remain unclear. In the present study, we investigated the effects of the combined administration of risedronate and menatetrenone on tacrolimus-induced bone loss in rats.

\section{Materials and Methods}

\subsection{Animals}

Four week-old male Wistar rats weighing 70-80 g were purchased from CLEA Japan Inc. (Tokyo, Japan). Animals were housed at $22 \pm 2{ }^{\circ} \mathrm{C}$ and $55 \pm 5 \%$ humidity on a 12-h light-dark cycle with ad libitum access to standard chow (MF; Oriental Yeast Co., Tokyo, Japan) and water. All procedures were approved by the Animal Research Committee of Niigata University of Pharmacy and Applied Life Sciences in accordance with the Japanese Government Animal Protection and Management Law, Japanese Government Notification on Feeding and Safekeeping of Animals.

\subsection{Drugs}

Commercially available tacrolimus (Astellas Pharma Inc., Tokyo, Japan) and risedronate (Eisai Co., Ltd., Tokyo, Japan) agents were obtained suspended in a $0.2 \%$ carboxymethylcellulose sodium solution (CMC-Na; Sigma-Aldrich, St. Louis, MO, USA). Menatetrenone (Eisai Co., Ltd., Tokyo, Japan) was suspended in olive oil.

\subsection{Experimental procedure}

Animals were randomly divided into four groups (10 animals/group): [1] control treated with the vehicle $(0.2 \% \mathrm{CMC}-\mathrm{Na})$, [2] tacrolimus at $1.5 \mathrm{mg} / \mathrm{kg}$, [3] tacrolimus at $1.5 \mathrm{mg} / \mathrm{kg}+$ risedronate at $1.0 \mathrm{mg} / \mathrm{kg}$, and [4] tacrolimus at $1.5 \mathrm{mg} / \mathrm{kg}+$ risedronate at $1.0 \mathrm{mg} / \mathrm{kg}$ + menatetrenone at $20 \mathrm{mg} / \mathrm{kg}$. Drug doses were selected based on previous reports relevant to tacrolimus (4), risedronate (12), and menatetrenone (27). Drugs were administered via oral gavage in a volume of $0.1 \mathrm{~mL} / 100$ $\mathrm{g}$ of body weight once daily for 4 weeks. All animals were euthanized under $\mathrm{CO}_{2}$ anesthesia $24 \mathrm{~h}$ after the final drug was administered. The femur and tibia were dissected, and soft tissue was removed.

\subsection{Bone strength analysis}

Bone strength of the femoral mid-diaphysis was evaluated via a three-point bending method using a mechanical testing machine (EZ-S; Shimadzu, Tokyo, Japan). The femur was positioned on two supports placed $10 \mathrm{~mm}$ apart. The bending load was vertically applied to the mid-diaphysis with a crosshead speed of $1.0 \mathrm{~mm} / \mathrm{min}$ until fracture. The load deformation curves were calculated using operation software (Trapezium $\mathrm{X}$; Shimadzu, Tokyo, Japan), and the maximum load, breaking energy, and stiffness were directly calculated from the load deformation curve.

\subsection{BMD measurements}

BMD of the whole femur and tibia was measured using quantitative computed tomography (LaTheta LCT-100; Aloka, Tokyo, Japan) with a pixel size of $250 \times 250 \mu \mathrm{m}$ and slice thickness of $1 \mathrm{~mm}$. The values of BMD were calculated using LaTheta software (ver. 1.31; Aloka, Tokyo, Japan).

\subsection{Bone histomorphometry}

We prepared non-decalcified specimens from the proximal tibia metaphysis according to the following method: The tibia was fixed with $70 \%$ ethanol for 7 days, stained with Villanueva Bone Stain (basic fuchsin, fast green, orange G, and azure II; Merck, Darmstadt, Germany) in $70 \%$ methanol for 7 days, and embedded in a methyl methacrylate resin. The resin blocks were then sliced to $5-\mu \mathrm{m}$ thickness on a microtome (Leica RM2255; Leica Inc., Nussloch, Germany). All bone histomorphometric parameters were measured at the secondary spongiosa region. To exclude the primary spongiosa, the measurement region was $0.42 .1 \mathrm{~mm}$ distal to the lowest point of the growth plate and $0.2 \mathrm{~mm}$ from the lateral cortex.

Bone histomorphometric measurements were performed using a semiautomatic image analyzing system (Histometry RT CAMERA; System Supply, Nagano, Japan) with $\times 400$ magnification. Bone structural parameters obtained included bone volume per tissue volume (BV/TV), trabecular thickness (Tb.Th), trabecular number (Tb.N), and trabecular separation (Tb. $\mathrm{Sp}$ ). Bone formation parameters included the osteoblast surface per bone surface (Ob.S/BS). Bone resorption parameters included the eroded surface per bone surface (ES/BS), osteoclast surface per bone surface (Oc.S/BS), 
and osteoclast number per bone surface (N.Oc/BS).

The dynamic parameter was measured using a double fluorescent labeling technique. For labeling, all rats were injected subcutaneously with $25 \mathrm{mg} / \mathrm{kg}$ of tetracycline (Sigma-Aldrich, St. Louis, MO, USA) and $10 \mathrm{mg} / \mathrm{kg}$ of calcein (Wako Pure Chemical Industries, Osaka, Japan) 5 and 2 days before they were euthanized, respectively. The labeled surface that reflected the calcification front at the time of tetracycline and calcein administration was visualized using a fluorescent microscope (Olympus BX50; Olympus America Inc., Center Valley, PA, USA). The parameters of single- and double-labeled surface (sLS and dLS) and inter-label thickness, and times (Ir. L.Th and Ir.L.t) were used in the calculation of the mineralizing surface per bone surface (MS [dLS+sLS/2]/ BS]), mineral apposition rate (MAR; Ir.L.Th/Ir.L.t).

Figure 1 shows the scheme of the primary parameters (28). Standard bone histomorphometrical nomenclature, symbols, and units were based on those described in the report of the American Society for Bone and Mineral Research Histomorphometry Nomenclature Committee (29).

\subsection{Statistical analysis}

Data are presented as the mean \pm standard error (SE). Differences between groups were analyzed by one-way ANOVA followed by Dunnett's multiple comparisons. $p$ $<0.05$ was considered significant.

\section{Results}

\subsection{Bone strength properties}

The maximum load and breaking energy in the tacrolimus group were significantly reduced $(37 \%$ and $40 \%$, respectively) compared with those in the control group (Figure 2). In addition, the maximum load and breaking energy in the risedronate and risedronate + menatetrenone groups were significantly higher

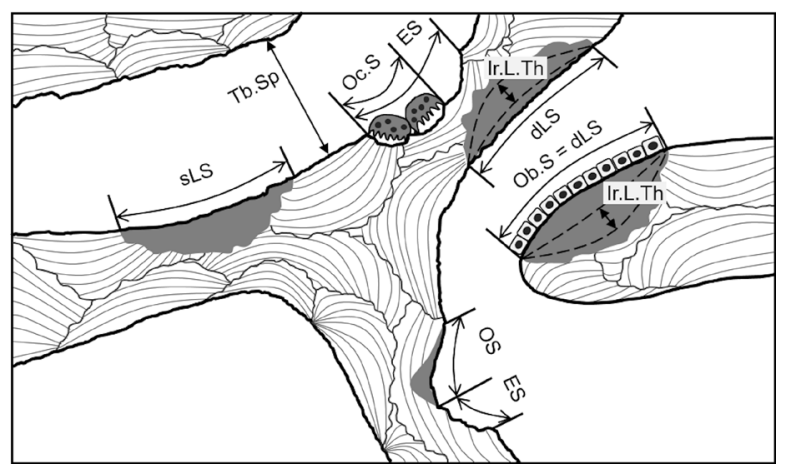

Figure 1. The scheme of the primary bone histomorphometric parameters. Tb.Sp; trabecular separation, dLS; double-labeled surface, sLS; single-labeled surface, Ir.L.Th; inter-labels thickness, Ob.S; osteoblast surface, OS; osteoid surface, ES; eroded surface, Oc.S; osteoclast surface. than those in the tacrolimus group. Furthermore, the maximum load and breaking energy in the risedronate + menatetrenone group were significantly higher than those in the risedronate group.

\subsection{BMD}

Femoral and tibial BMD were significantly reduced ( $9 \%$ and $11 \%$, respectively) in the tacrolimus group compared with those in the control group (Figure 3 ). The BMD of femur and tibia in the risedronate and risedronate + menatetrenone groups were significantly higher than those in the tacrolimus group. On the other hand, the BMD of femur and tibia in the risedronate + menatetrenone group were comparable with and not significantly different from those in the risedronate group.

\subsection{Bone histomorphometric evaluation}

Trabecular bone structural parameters according to the bone histomorphometry of the proximal tibia metaphysis are shown in Figure 4. Tacrolimus treatment significantly reduced the BV/TV, Tb.Th, and Tb.N (50\%, $20 \%$, and $66 \%$, respectively), and increased the Tb.SP $(57 \%)$ relative to those in the control group. These bone structural parameters in the risedronate and risedronate + menatetrenone groups were significantly higher than those in the tacrolimus group.

Bone formation parameters, Ob.S/BS, MS/BS, and MAR, in the tacrolimus group did not significantly differ from those in the control group, whereas bone resorption parameters, ES/BS, Oc.S/BS, and N.Oc/BS (69\%, 70\%, and $87 \%$, respectively), were significantly increased (Figure 5). The tacrolimus-induced increases in ES/BS, Oc.S/BS, and N.Oc/BS were significantly suppressed by risedronate alone or risedronate + menatetrenone. However, Ob.S/BS, MS/BS, and MAR (83\%, 70\%, and $54 \%$, respectively), were significantly reduced in the risedronate group compared with the other groups. In contrast, Ob.S/BS, MS/BS, and MAR were significantly increased in the risedronate + menatetrenone group compared with those in the risedronate group, and were comparable with those in the control group.

Typical fluorescence microphotographs of the slices assessed by bone histomorphometry are shown in Figure 6 . These images confirmed the marked decrease in fluorescence labeled surface with tetracycline and calcein in the risedronate group compared with that in the control group. In contrast, bones in the risedronate + menatetrenone group had a marked increase in fluorescence labeled surface and inter-label thickness compared with those in the risedronate group.

\section{Discussion}

The present study examined the effects of the combined 

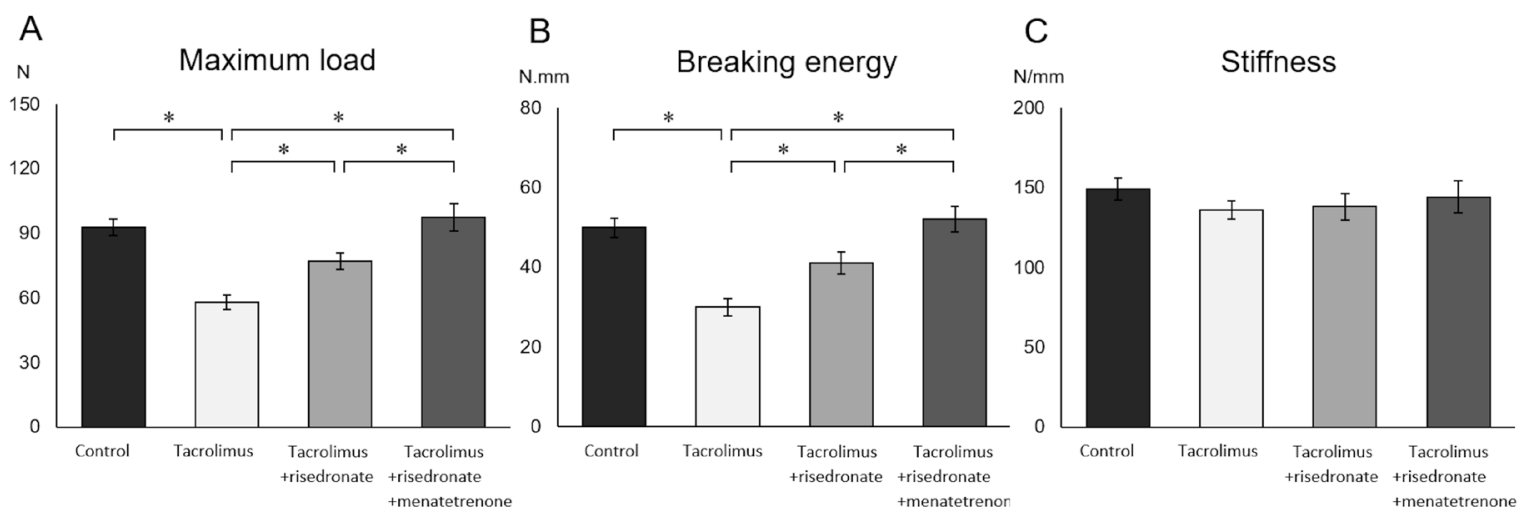

Figure 2. Bone strength properties (A: maximum load, B: breaking energy, C: stiffness) of the femoral mid-diaphysis in each group. Bone strength was evaluated by a three-point bending method. Data represents the mean $\pm \mathrm{SE}(n=10) .{ }^{*} p<0.05$.
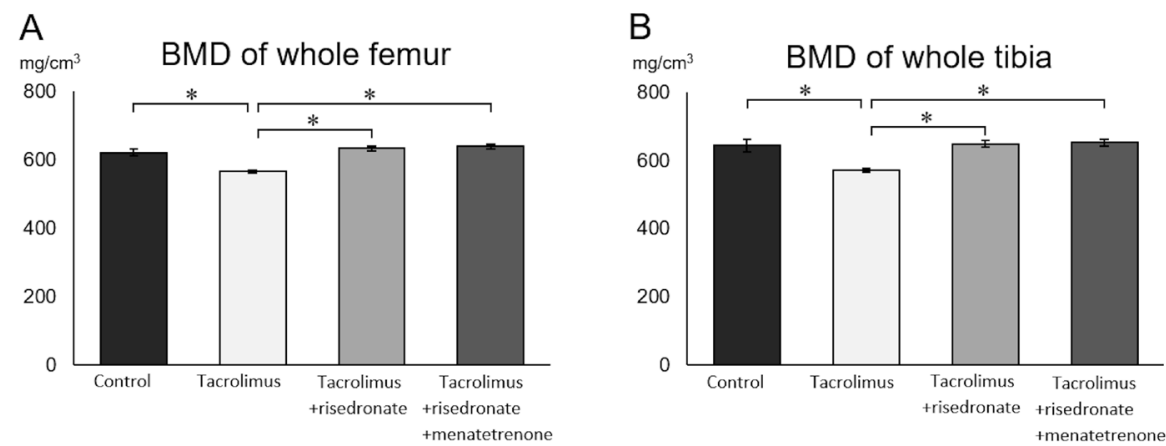

Figure 3. BMD of whole femur (A) and tibia (B) in each group. BMD was measured using quantitative computed tomography. Data represents the mean $\pm \mathrm{SE}(n=10) .{ }^{*} p<0.05$.

A

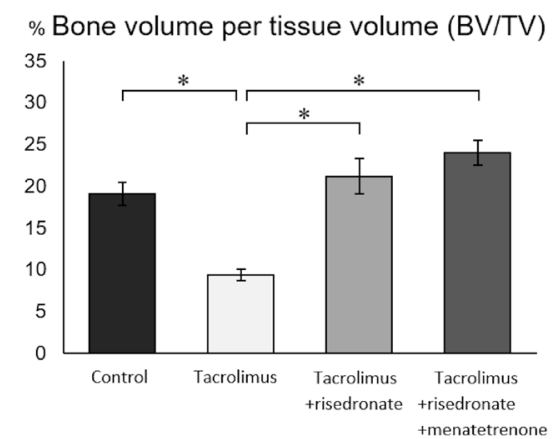

C

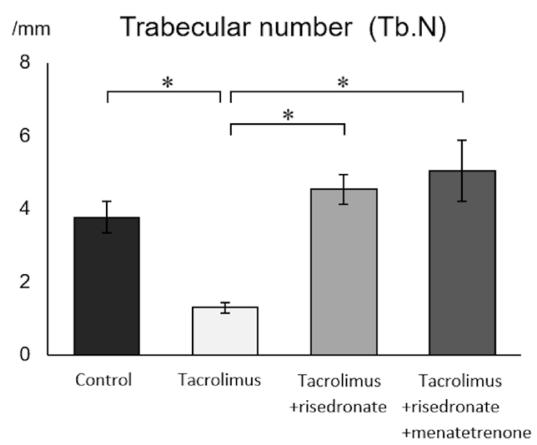

B

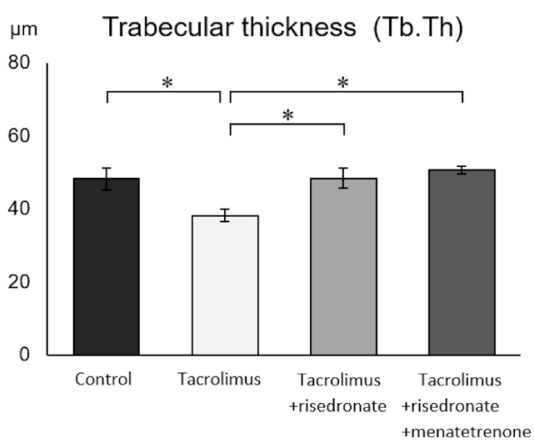

D



Figure 4. Trabecular bone structural parameters (A: bone volume per tissue volume (BV/TV), B: trabecular thickness (Tb.Th), C: trabecular number (Tb.N), D: trabecular separation (Tb.Sp)) according to the bone histomorphometry of the proximal tibia metaphysis. Data represents the mean $\pm \mathrm{SE}(n=10) .{ }^{*} p<0.05$. 

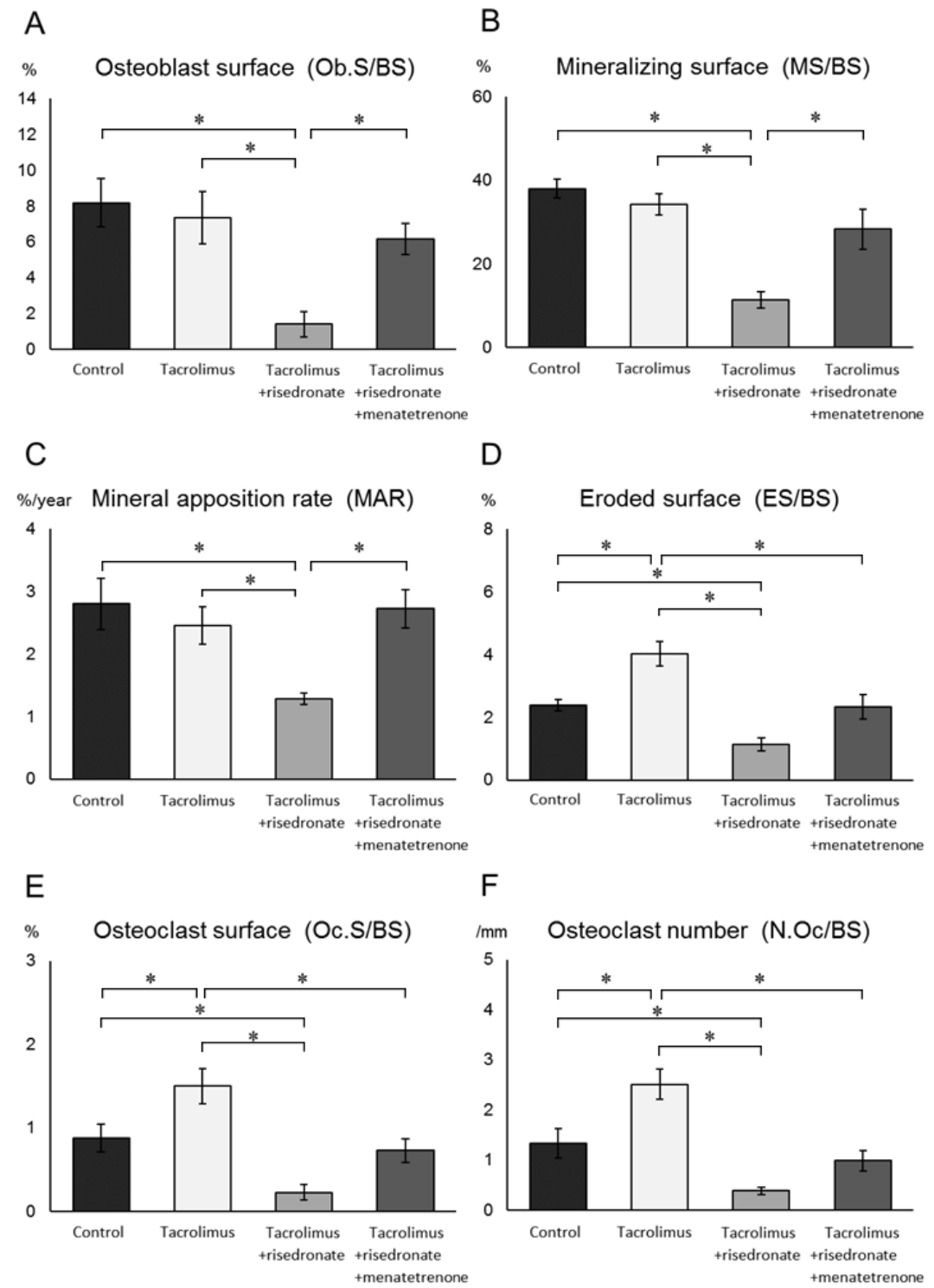

Figure 5. Bone formation parameters (A: osteoblast surface (Ob.S/BS), B: mineralizing surface (MS/BS), C: mineral apposition rate (MAR), D: eroded surface (ES/BS), E: osteoclast surface (Oc.S/BS), F: osteoclast number (N.Oc/BS)) according to bone histomorphometry of the proximal tibia metaphysis. Data represents the mean $\pm \operatorname{SE}(n=10) . " p<0.05$.

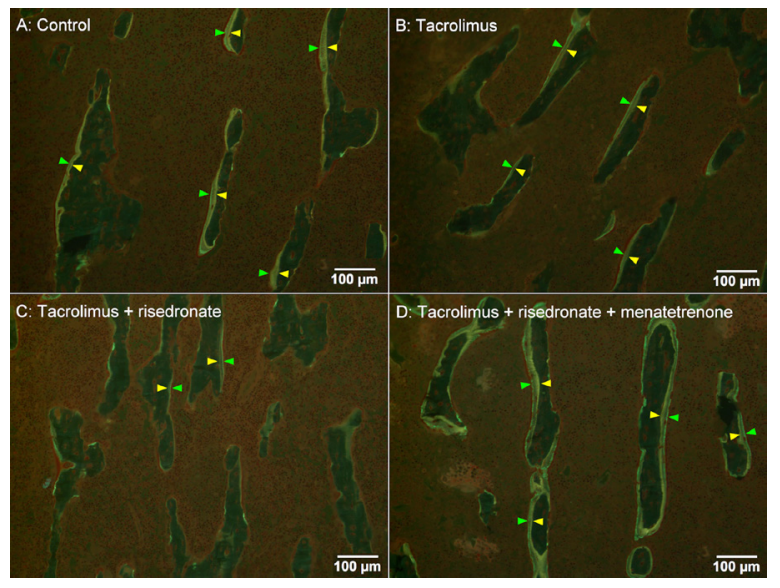

Figure 6. Typical micrographs of the slices assessed by bone histomorphometry under fluorescence. The labeling surface with tetracycline and calcein is indicated by the yellow arrows and the green arrows, respectively. administration of risedronate and menatetrenone on tacrolimus-induced bone loss. The administration of tacrolimus at $1.5 \mathrm{mg} / \mathrm{kg} /$ day for 4 weeks significantly reduced the femoral and tibial $\mathrm{BMD}$ in rats. The tacrolimus-induced decreases in the BMD of the lower limb bones were significantly suppressed by risedronate alone, although no additive effects were achieved by the combined administration of risedronate and menatetrenone. On the other hand, the three-point bending test of bone strength properties demonstrated that risedronate was more effective in preventing and ameliorating the tacrolimus-induced decrease in bone strength properties when combined with menatetrenone than when administered alone.

Only a few studies have examined the effects of anti-osteoporosis drugs on tacrolimus-induced bone loss. The clinical studies $(30,31)$ and animal 
studies $(32,33)$ found significant protective effects of bisphosphonate on calcineurin inhibitor-induced bone loss. However, long-term administration of bisphosphonate increases microdamage accumulation in the bone (15-17) and is involved in the development of atypical femoral fractures (18-21). Specifically, the marked suppression of bone turnover by longterm bisphosphonate administration may degrade bone quality, resulting in bone fragility. To prevent the bone damage induced by immunosuppressive agents, care should be exercised not only to increase BMD, but also to improve bone quality.

Several studies using osteoporosis animal models also reported the positive effects of the combined administration of bisphosphonate and menatetrenone on bone metabolism $(34,35)$. Menatetrenone is expected to reduce the risk of fracture primarily by improving bone quality (27) because of its negligible bone massincreasing effects (26). Menatetrenone may improve the degradation of bone quality induced by bisphosphonate. Therefore, in the present study, in order to evaluate the bone quality, we performed a bone histomorphometry of the proximal tibia metaphysis. Bone histomorphometric analysis revealed the impaired microstructure of trabecular bone in rats administered tacrolimus. It also significantly increased the ES/BS, Oc.S/BS, and N.Oc/ BS without significantly affecting bone formation. Thus, as previously reported (4), tacrolimus caused rarefaction of the trabecular bone by increasing bone resorption. Risedronate alone significantly suppressed the tacrolimus-induced increase in bone resorption and significantly reduced the bone formation parameters, Ob.S/BS, MS/BS, and MAR. MS/BS and MAR are considered an index of osteoblast differentiation and proliferation (36). Of note, Ob.S/BS, MS/BS, and MAR were significantly higher in the risedronate + menatetrenone group than in the control group. Thus, the suppression of bone formation by risedronate was significantly prevented and ameliorated by the combined administration of risedronate and menatetrenone. Menatetrenone promotes osteocalcin production and mineralization in osteoblasts $(37,38)$, and suppresses osteoclast formation and bone resorption in vitro (39). The additive improving effects of the combined administration of risedronate and menatetrenone on the tacrolimus-induced decrease in bone strength may be partially explained by the menatetrenone-induced enhancement of bone formation.

In conclusion, the present study investigated the effectiveness of the combined administration of risedronate and menatetrenone against tacrolimusinduced bone loss. Risedronate alone and risedronate + menatetrenone significantly improved tacrolimusinduced decreases in bone strength properties and BMD. Moreover, the combined administration of risedronate and menatetrenone was highly effective in improving the tacrolimus-induced decrease in bone strength properties. The effectiveness of the combined administration of anti-osteoporosis drugs with different mechanisms of action may be caused by menatetrenone, which ameliorated the marked suppression of bone formation due to bisphosphonates.

\section{References}

1. Raisz LG. Physiology and pathophysiology of bone remodeling. Clin Chem. 1999; 45:1353-1358.

2. Manolagas SC. Birth and death of bone cells: basic regulatory mechanisms and implications for the pathogenesis and treatment of osteoporosis. Endocr Rev. 2000; 21:115-137.

3. Rodan GA, Martin TJ. Therapeutic approaches to bone diseases. Science. 2000; 289:1508-1514.

4. Kanda J, Izumo N, Furukawa M, Shimakura T, Yamamoto N, E Takahashi H, Asakura T, Wakabayashi H. Effects of the calcineurin inhibitors cyclosporine and tacrolimus on bone metabolism in rats. Biomed Res (Tokyo). 2018; 39:131-139.

5. NIH Consensus Development Panel on Osteoporosis Prevention, Diagnosis, and Therapy. Osteoporosis prevention, diagnosis, and therapy. JAMA. 2001; 285:785795.

6. Russell RG, Croucher PI, Rogers MJ. Bisphosphonates: pharmacology, mechanisms of action and clinical uses. Osteoporos Int. 1999; 9:S66-80.

7. van Beek ER, Cohen LH, Leroy IM, Ebetino FH, Löwik CW, Papapoulos SE. Differentiating the mechanisms of antiresorptive action of nitrogen containing bisphosphonates. Bone. 2003; 33:805-811.

8. Eastell R, Walsh JS, Watts NB, Siris E. Bisphosphonates for postmenopausal osteoporosis. Bone. 2011; 49:82-88.

9. Harris ST, Watts NB, Genant HK, McKeever CD, Hangartner T, Keller M, Chesnut CH 3rd, Brown J, Eriksen EF, Hoseyni MS, Axelrod DW, Miller PD. Effects of risedronate treatment on vertebral and nonvertebral fractures in women with postmenopausal osteoporosis: a randomized controlled trial. Vertebral Efficacy With Risedronate Therapy (VERT) Study Group. JAMA. 1999; 282:1344-1352.

10. Mortensen L, Charles P, Bekker PJ, Digennaro J, Johnston CC Jr. Risedronate increases bone mass in an early postmenopausal population: two years of treatment plus one year of follow-up. J Clin Endocrinol Metab. 1998; 83:369-402.

11. Reginster J, Minne HW, Sorensen OH, Hooper M, Roux C, Brandi ML, Lund B, Ethgen D, Pack S, Roumagnac I, Eastell R. Randomized trial of the effects of risedronate on vertebral fractures in women with established postmenopausal osteoporosis. Vertebral Efficacy with Risedronate Therapy (VERT) Study Group. Osteoporos Int. 2000; 11:83-91.

12. Erben RG, Mosekilde L, Thomsen JS, Weber K, Stahr K, Leyshon A, Smith SY, Phipps R. Prevention of bone loss in ovariectomized rats by combined treatment with risedronate and 1alpha, 25-dihydroxyvitamin D3. J Bone Miner Res. 2002; 17:1498-1511.

13. Ito M, Nakayama K, Konaka A, Sakata K, Ikeda K, Maruyama T. Effects of a prostaglandin EP4 agonist, ONO-4819, and risedronate on trabecular microstructure and bone strength in mature ovariectomized rats. Bone. 
2006; 39:453-459.

14. Ito M, Nishida A, Aoyagi K, Uetani M, Hayashi K, Kawase M. Effects of risedronate on trabecular microstructure and biomechanical properties in ovariectomized rat tibia. Osteoporos Int. 2005; 16:10421048.

15. Li J, Mashiba T, Burr DB. Bisphosphonate treatment suppresses not only stochastic remodeling but also the targeted repair of microdamage. Calcif Tissue Int. 2001; 69:281-286.

16. Mashiba T, Hirano T, Turner CH, Forwood MR, Johnston CC, Burr DB. Suppressed bone turnover by bisphosphonates increases microdamage accumulation and reduces some biomechanical properties in dog rib. J Bone Miner Res. 2000; 15:613-620.

17. Mashiba T, Turner CH, Hirano T, Forwood MR, Johnston CC, Burr DB. Effects of suppressed bone turnover by bisphosphonates on microdamage accumulation and biomechanical properties in clinically relevant skeletal sites in beagles. Bone. 2001; 28:524-531.

18. Capeci CM, Tejwani NC. Bilateral low-energy simultaneous or sequential femoral fractures in patients on long-term alendronate therapy. J Bone Joint Surg Am. 2009; 91:2556-2561.

19. Odvina CV, Zerwekh JE, Rao DS, Maalouf N, Gottschalk FA, Pak CY. Severely suppressed bone turnover: a potential complication of alendronate therapy. J Clin Endocrinol Metab. 2005; 90:1294-1301.

20. Park-Wyllie LY, Mamdani MM, Juurlink DN, Hawker GA, Gunraj N, Austin PC, Whelan DB, Weiler PJ, Laupacis A. Bisphosphonate use and the risk of subtrochanteric or femoral shaft fractures in older women. JAMA. 2011; 305:783-789.

21. Patel VC, Lazzarini AM. Bilateral simultaneous femoral diaphyseal fractures in a patient with long-term ibandronate use. Orthopedics. 2010; 33:775.

22. Shearer MJ. Vitamin K. Lancet. 1995; 345:229-234.

23. Vermeer C, Jie KS, Knapen MH. Role of vitamin K in bone metabolism. Annu Rev Nutr. 1995; 15:1-22.

24. Ishida Y, Kawai S. Comparative efficacy of hormone replacement therapy, etidronate, calcitonin, alfacalcidol, and vitamin $\mathrm{K}$ in postmenopausal women with osteoporosis: the Yamaguchi osteoporosis prevention study. Am J Med. 2004; 117:549-555.

25. Shiraki M, Shiraki Y, Aoki C, Miura M. Vitamin $\mathrm{K}_{2}$ (menatetrenone) effectively prevents fractures and sustains lumbar bone mineral density in osteoporosis. J Bone Miner Res. 2000; 15:515-521.

26. Kobayashi M, Hara K, Akiyama Y. Infrared analysis of bones in magnesium-deficient rats treated with vitamin $\mathrm{K}_{2}$. J Bone Miner Metab. 2007; 25:12-18.

27. Hara K, Akiyama Y. Collagen-related abnormalities, reduction in bone quality, and effects of menatetrenone in rats with a congenital ascorbic acid deficiency. J Bone Miner Metab. 2009; 273:324-332.

28. Takahashi EH. Histomorphometric parameters of trabecular bone. In: Handbook of Bone Morphometry (Takahashi EH, eds.). Nishimura Co. Ltd., Niigata, Japan, 1997; pp. 76-82.
29. Dempster DW, Compston JE, Drezner MK, Glorieux FH, Kanis JA, Malluche H, Meunier PJ, Ott SM, Recker RR, Parfitt AM. Standardized nomenclature, symbols, and units for bone histomorphometry: a 2012 update of the report of the ASBMR Histomorphometry Nomenclature Committee. J Bone Miner Res. 2013; 28:2-17.

30. Fan SL, Kumar S. Cunningham J. Long-term effects on bone mineral density of pamidronate given at the time of renal transplantation. Kidney Int. 2003; 63:2275-2279.

31. Grotz W, Nagel C, Poeschel D, Cybulla M, Petersen KG, Uhl M, Strey C, Kirste G, Olschewski M, Reichelt A, Rump LC. Effect of ibandronate on bone loss and renal function after kidney transplantation. J Am Soc Nephrol. 2001; 12:1530-1537.

32. Matsunaga T, Shigetomi M, Hashimoto T, Suzuki H, Gondo T, Tanaka H, Sugiyama T, Taguchi T. Effects of bisphosphonate treatment on bone repair under immunosuppression using cyclosporine $\mathrm{A}$ in adult rats. Osteoporos Int. 2007; 18:1531-1540.

33. Zeni SN, Gregorio S, Gomez AC, Somoza J, Mautalen C. Olpadronate prevents the bone loss induced by cyclosporine in the rat. Calcif Tissue Int. 2002; 70:48-53.

34. Iwasaki Y, Yamato H, Murayama H, Sato M, Takahashi T, Ezawa I, Kurokawa K, Fukagawa M. Combination use of vitamin $\mathrm{K}_{2}$ further increases bone volume and ameliorates extremely low turnover bone induced by bisphosphonate therapy in tail-suspension rats. J Bone Miner Metab. 2003; 21:154-160.

35. Sasaki H, Miyakoshi N, Kasukawa Y, Maekawa S, Noguchi H, Kamo K, Shimada Y. Effects of combination treatment with alendronate and vitamin $\mathrm{K}_{2}$ on bone mineral density and strength in ovariectomized mice. $\mathrm{J}$ Bone Miner Metab. 2010; 28:403-409.

36. Recker RR, Kimmel DB, Dempster D, Weinstein RS, Wronski TJ, Burr DB. Issues in modern bone histomorphometry. Bone. 2011; 49:955-964.

37. Koshihara $Y$, Hoshi K, Ishibashi $H$, Shiraki M. Vitamin $\mathrm{K}_{2}$ promotes $1 \alpha, 25(\mathrm{OH})_{2}$ vitamin $\mathrm{D}_{3}$-induced mineralization in human periosteal osteoblasts. Calcif Tissue Int. 1996; 59:466-473.

38. Tabb MM, Sun A, Zhou C, Grün F, Errandi J, Romero K, Pham H, Inoue S, Mallick S, Lin M, Forman BM, Blumberg B. Vitamin $\mathrm{K}_{2}$ regulation of bone homeostasis is mediated by the steroid and xenobiotic receptor SXR. J Biol Chem. 2003; 278:43919-43927.

39. Hara K, Akiyama Y, Nakamura T, Murota S, Morita I. The inhibitory effect of vitamin $\mathrm{K}_{2}$ (menatetrenone) on bone resorption may be related to its side chain. Bone. 1995; 16:179-184.

Received April 3, 2020; Revised April 24, 2020; Accepted April 27, 2020

*Address correspondence to:

Dr. Hiroyuki Wakabayashi, Department of Clinical Pharmacotherapy, Faculty of Pharmaceutical Sciences, Niigata University of Pharmacy and Applied Life Sciences, 265-1 Higashijima, Akiha-ku, Niigata 956-8603, Japan.

E-mail:waka@nupals.ac.jp 